Original article

\title{
Mobility in association with anxiety and quality of life in middle-aged and older female fallers and non-fallers
}

\author{
Taisiya P. Shiryaeva ${ }^{1,2}$, Denis M. Fedotov ${ }^{1,3}$, Anatoliy V. Gribanov ${ }^{1,2}$, Mikhail N. Pankov ${ }^{1}$, Larisa F. Startseva ${ }^{4}$, \\ Sergey F. Bagretsov ${ }^{1}$ \\ ${ }^{1}$ Northern (Arctic) Federal University, Arkhangelsk, Russia \\ ${ }^{2}$ Institute of Age Physiology, Moscow, Russia \\ ${ }^{3}$ Northern State Medical University, Arkhangelsk, Russia \\ ${ }^{4}$ State Institution of Pharmaceutical Drugs and Good Practices, Moscow, Russia
}

Received 31 Octoberl 2019, Revised 2 March 2020, Accepted 23 April 2020

(C) 2019, Shiryaeva T.P., Fedotov D.M., Gribanov A.V., Pankov M.N., Startseva L.F., Bagretsov S.F.

(C) 2019, Russian Open Medical Journal

\begin{abstract}
Objective - The goal of our study was to compare mobility vs. cognitive function and quality of life in middle-aged and older women, both fallers and non-fallers.

Material and Methods - A total of 246 healthy community-dwelling women 55-74 YO participated in this study. They were distributed among two subgroups: (1) fallers $(n=95)$ and (2) non-fallers $(n=151)$. Fallers had falls in the past 12 months, while non-fallers did not. Walking tests were performed using Balance Master ${ }^{\circledR}$ posturography system. Trait anxiety and state anxiety were assessed by SpielbergerHanin test; health state and quality of life (QoL) were evaluated from SF-36 health survey questionnaire; subjectively experienced age (SEA) and the rate of aging were also estimated.

Results - In Walk Across test, the average speed was lower in fallers ( $p=0.020)$, as compared with non-fallers. In Tandem Walking test, step width $(p=0.016)$ and endpoint sway velocity $(p=0.029)$ were higher, while speed $(p=0.030)$ was lower in fallers vs. non-fallers. StepQuick-Turn test time $(p=0.016)$ was longer and sway $(p=0.011)$ was higher in fallers in the course of turning both to the left and to the right. SEA in female non-fallers was less $(p=0.033)$ than in female fallers of the same age. Trait anxiety was higher in female fallers $(p=0.012)$ with a lower QoL level $(p<0.001)$.

Conclusion - Our results validated the need for early assessment of the postural control dynamic components in order to prevent the development of falls as a geriatric syndrome.
\end{abstract}

Keywords: mobility, walking, aging, falling, anxiety, quality of life.

Cite as Shiryaeva TP, Fedotov DM, Gribanov AV, Pankov MN, Startseva LF, Bagretsov SF. Mobility in association with anxiety and quality of life in middle-aged and older female fallers and non-fallers. Russian Open Medical Journal 2020; 9: e0212.

Correspondence to Taisiya P. Shiryaeva. E-mail: taisia.moroz@yandex.ru.

\section{Introduction}

It is acknowledged that age-related changes in postural control are common among older people, causing their falls. The latter represent danger at older age and can result in serious injuries, loss of social independence, and even premature death [1-2]. Recent studies of this issue demonstrated aging peculiarities in elderly and senile people, particularly falls as a geriatric syndrome. Similar scientific research identified the characteristics of static and dynamic components in a postural control as typical concerns in geriatric medicine, and measured the parameters of these changes in predicting the development of falls as a geriatric syndrome [3-6]. The aging of human body is a very individual process, since it is influenced by multiple factors. There are several important events occurring with age: development of cardiovascular diseases; decrease in the number of acute illnesses and increase in the number of ailments associated with progression of chronic pathological processes; decline in muscle strength and mass; reduction in bone density. As a consequence, we observe a decline in postural control functions, which is a clear manifestation of reduced mobility, resulting in possibility of falls [7-8].

The Kellogg International Work Group on the Prevention of Falls by the Elderly defined fall as an incident, in which an individual suddenly finds himself or herself on the ground or other low surface, except in cases arising from strokes, loss of consciousness, sudden paralysis or epileptic seizures [9]. It is wellknown that 50 to $70 \%$ of the falls in elderly and senile age people occur during walking. Gait posturography is used to investigate the balance in the vertical body position. It is the method of detecting the posture and projection of a gravity center displacement onto the base of a posturography support platform. The position of a gravity center projection; characteristics of vibrations, such as their amplitude, frequency and direction; and the average posture in the projection onto the base of a support platform are sensitive parameters reflecting the status of various systems involved in the 
process of maintaining the balance [10-11]. Studies of gait parameters in elderly people are becoming an increasingly practical application in gerontology and geriatrics, since they facilitate predicting the risk of falls and degree of mobility deterioration [11]. It was noted that rehabilitation programs aimed at preserving the dynamic components of postural control in the patients with postural instability had a positive impact on reducing the risk of falls and subsequent safeguarding of mobility [12].

Walk Across, Tandem Walking, and Step-Quick-Turn tests are customary used for identifying the parameters of a walking gait, which allows predicting the risk of falls and reduced mobility, as well as for quality assessment and correction of rehabilitation programs related to the changes in a postural control. In addition, participation in sports and recreational activities for people aged 65 and older with a "falls syndrome" helps preserving their abilities to complex coordination movements and complex motor acts, has a positive impact on their mobility and social independence, and reduces the risk of further falls. All of the above prolong their longevity and improve the quality of their lives $[2,13]$. The objective of our study was to compare mobility and cognitive characteristics among middle-aged and older women, who had falls during the last 12 months, and non-fallers serving as a gender-matched control.

\section{Material and Methods}

\section{Participants}

The study enrolled 246 healthy community-dwelling women 55-74 YO who volunteered to participate. The participants were informed of the goal and potential risks of the study and signed written informed consent in accordance with the outlines of the Declaration of Helsinki by the World Medical Association, Ethical Principles for Medical Research Involving Human Subjects. This subject pool was split into two groups: fallers ( $n=95$, mean age: 64.7 yo, SD: 5.28 yr) and non-fallers ( $n=151$, mean age: 64.5 yo, SD: $4.08 \mathrm{yr}$ ) (Table 1). Fallers had two or more falls during the last 12 months prior to examination. The study criteria excluded women with mental disabilities, stroke in anamnesis, cognitive impairment, chronic traumas and those residing in nursing homes. In addition, all women at the time of survey were mobile and able to walk without assistance. The research was conducted during 2016-2017. Informed consent was obtained from all individual participants included in the study.

Table 1 Data on female fallers and non-fallers, mean (SD)

\begin{tabular}{lccc}
\hline Parameters & Fallers $(n=95)$ & Non-fallers $(n=151)$ & $P$ \\
\hline Age $($ YO) & $64.7(5.3)$ & $64.5(4.1)$ & 0.787 \\
Height $(\mathrm{cm})$ & $157.4(5.8)$ & $157.4(5.7)$ & 0.918 \\
Body mass (kg) & $75.6(13.7)$ & $74.0(13.5)$ & 0.360 \\
BMI (kg/m ${ }^{2}$ ) & $30.6(5.2)$ & $29.9(4.9)$ & 0.322 \\
Falls (times per 12 months) & $1.1(0.2)$ & - & - \\
SEA (yr.) & $60.9(6.6)$ & $59.0(6.4)^{*}$ & 0.033 \\
Rate of aging (yr.) & -6.2 & $-8.4^{* *}$ & 0.005 \\
State anxiety (pts) & $41.2(10.1)$ & $40.1(8.5)$ & 0.366 \\
Trait anxiety (pts) & $49.5(7.6)$ & $47.0(7.7)^{*}$ & 0.012 \\
PhHCQOL (pts) & $59.9(19.5)$ & $69.8(16.7)^{* * *}$ & 0.000 \\
PsHCQOL (pts) & $61.7(19.9)$ & $71.0(17.9)^{* * *}$ & 0.000 \\
MCQOL (pts) & $60.8(19.7)$ & $70.4(17.3)^{* * *}$ & 0.000 \\
\hline
\end{tabular}

SEA, subjectively experienced age; BMI, body mass index; PhHCQOL, physical health quality of life component; PsHCQOL, psychological health quality of life component; MCQOL, mean quality of life component. Fallers had two or more falls in the last 12 months prior to examination.

${ }^{*} p<0.05 ;{ }^{* *} p<0.01 ;{ }^{* * *} p<0.001$ compared to fallers.

\section{Experimental setup}

Mobility assessment. The Balance Master ${ }^{\circledR}$ computerized posturography system (USA) including a fixed $45.72 \mathrm{~cm} \times 152.4 \mathrm{~cm}$ force plate was used to measure the forces, exerted through the patient's feet, and to calculate the center of gravity (COG) position and postural control characteristics. The long force plate allowed enhancing assessment capabilities. In order to assess postural control in older women, sit-to-stand test and different walking tests were conducted.

Sit-to-stand (STS) assessment quantifies the patient's ability to rise from a seated mode to a standing position. Key components of this task included shifting the body COG forward from an initial position over the seat to a location centered over the base of support, followed by body extension into upright position, while maintaining centered COG position. The measured parameters were weight transfer time, rising index (the vertical force exerted by the legs during the rising phase), sway velocity during the rising phase, and left/right symmetry of the rising force.

Walking tests assessment. The following tests were used:

Walk Across (WA) test quantified characteristics of gait while patients walked across the length of the force plate. The test characterized steady state gait by having the patient begin well behind and continuing beyond the force plate. Measured parameters were average step width, average step length, speed, and step length symmetry.

Tandem Walking (TW) test characterized numerically the gait traits as the patient walked heel to toe from one end of the force plate to another. Measured parameters were step width, speed, and endpoint sway velocity.

Step-Quick-Turn (SQT) test was measuring turn performance characteristics, as the patients were taking two forward steps, quickly turning $180^{\circ}$, and returning to the starting point. Measured parameters were: the time necessary to execute the turn, and sway velocity during the turn execution.

Step-Up-and-Over (SUO) test calculated motor control characteristics, as the patient stepped up onto a curb with one foot, swung the other foot over the curb while lifting the body through upright standing position as quickly as possible, and then lowered the body weight to land the swing leg on the force plate as gently as possible. Measured parameters were lift-up index (force to rise), movement time, and impact index (control of impact force descending onto the swing leg).

Anxiety assessment. Anxiety is among major factors contributing to the development of human mental and physical ailments, which could lead to the "falls syndrome". The anxiety level has been studied by self-assessment scales of both state anxiety and trait anxiety sensu Spielberger-Hanin [7; 14]. The following levels of anxiety were numerically specified by the score (total points): low $(<30)$, medium (31-44) and high (>45).

Quality of life assessment. To assess the quality of life (QoL), the SF-36 health survey questionnaire was used. The questionnaire was designed to study all components of QoL, including those related to health and not specific to age groups, certain ailments or treatment programs. For convenience, all QoL components assessed by SF-36 scales were grouped into two indicators:

(1) The physical health component of quality of life (PhHCQOL). The scale includes scoring for physical functioning, overall state of health, role functioning based on physical condition, and pain intensity; 
(2) The psychological health component of quality of life (PsHCQOL). The scale includes scoring for mental health, life activity, social functioning, and role functioning based on emotional state.

We have also calculated the mean component of quality of life (MCQOL) by computing the average of PhHCQOL and PsHCQOL.

Assessment of subjectively experienced age and rate of aging. Subjectively experienced age (SEA) in middle-aged and older women was determined by asking the respondents about how old did they feel they were. This parameter values depended on the patients' life intensity, eventfullness and satisfaction; experience, perceived degree of self-realization and socio-economic status. For calculating the rate of aging, we used the algorithms for determining the biological age (BA) and appropriate biological age (ABA) via V.P. Voitenko techniques. The magnitude of the rate of aging (RA) was determined according to the formula: $R A=B A-A B A$ [8].

\section{Statistical analysis}

The analyses were performed using IBM $^{\circledR}$ SPSS ${ }^{\circledR}$ Statistics version 23.0 (USA). The normal distribution of the measured variables was assessed using the Shapiro-Wilk test. Standard statistical methods were employed for calculation of means and standard deviations of the mean $( \pm S D)$. Differences between the study subjects were examined using a Student's $t$-test. Statistical significance was set at $p<0.05$.

\section{Results}

Data of anthropometry, anxiety, RA and QoL are presented in Table 1.

There were no significant differences in age and measured anthropometric traits between fallers and non-fallers. In both groups of women, SEA was lower than their actual age. However, this indicator was lower in non-fallers compared to fallers $(p=0.033)$. Comparative evaluation of the RA showed that in female fallers this parameter was lower than in non-fallers $(p=0.005)$. The analysis of state anxiety by Spielberger - Hanin method did not find statistically significant differences between the groups $(p=0.366)$. Comparative characteristics of trait anxiety by Spielberger-Hanin yielded lower values in fallers, as compared with non-fallers $(p=0.012)$. While analyzing QoL, we found that values of PhHCQOL $(p<0.001)$, PSHCQOL $(p<0.001)$ and MCQOL $(p<0.001)$ were lower in fallers vs. non-fallers.

Characteristics of mobility in female fallers and non-fallers are given in Table 2. No significant differences between measured groups were implied by STS test parameters, as well as by SUO test movement time and impact index (Table 2).

Also, we did not discover significant differences in step widths and lengths in WA test between fallers and non-fallers. The walking speed in WA test was lower in fallers $(p=0.026)$ compared with non-fallers. TW test showed statistically significant higher step width $(p=0.016)$ and endpoint sway $(p=0.029)$, and significantly lower speed $(p=0.030)$ between the measured groups. The results of SQT test suggested that, in fallers, time $(p=0.016)$ was longer and sway value during turning left or right was higher $(p=0.011)$, compared with non-fallers. At the same time, SUO test revealed that lift-up index values were higher in non-fallers than in fallers $(p=0.008)$.
Table 2 Characteristics of mobility in female fallers and non-fallers, mean (SD)

\begin{tabular}{lccc}
\hline Parameters & Fallers $(n=95)$ & Non-fallers $(n=151)$ & $P$ \\
\hline Weight transfer time, STS (s) & $0.5(0.2)$ & $0.5(0.2)$ & 0.416 \\
Rising index, STS (cm/s) & $17.7(6.8)$ & $18.3(6.7)$ & 0.541 \\
Sway velocity, STS $(\mathrm{cm} / \mathrm{s})$ & $3.8(1.0)$ & $3.6(1.1)$ & 0.058 \\
Step width, WA $(\mathrm{cm})$ & $13.2(3.1)$ & $13.2(3.2)$ & 0.993 \\
Step length, WA (cm) & $55.8(12.1)$ & $58.3(9.8)$ & 0.077 \\
Speed, WA (cm/s) & $82.1(14.3)$ & $85.9(12.2)^{*}$ & 0.026 \\
Step width, TW (cm) & $8.3(2.6)$ & $7.6(1.9)^{*}$ & 0.016 \\
Speed, TW (cm/s) & $28.6(7.9)$ & $30.9(8.1)^{*}$ & 0.030 \\
Endpoint sway, TW (cm/s) & $5.8(1.8)$ & $5.3(1.8)^{*}$ & 0.029 \\
Time, SQT (s) & $1.7(0.8)$ & $1.5(0.9)^{*}$ & 0.011 \\
Sway, SQT (cm/s) & $29.8(9.5)$ & $26.4(10.5)^{*}$ & 0.016 \\
Lift-up index, SUO (cm/s) & $39.5(11.1)$ & $43.8(11.8)^{* *}$ & 0.008 \\
Movement time, SUO (s) & $1.6 \pm 0.5)$ & $1.5 \pm 0.4$ & 0.136 \\
Impact index, SUO (cm/s) & $61.0 \pm 24.2)$ & $60.3 \pm 23.1$ & 0.833 \\
\hline
\end{tabular}

STS, Sit-to-stand test; WA, Walk Across test; TW, Tandem Walking test; SQT, Step-Quick-Turn test; SUO, Step-Up-and-Over test.

${ }^{*} p<0.05{ }^{* *} p<0.01 ;{ }^{* * *} p<0.001$ compared to fallers.

\section{Discussion}

The main findings of our study were: (1) temporal and spatial characteristics of gait in female fallers 55-74 YO were markedly lower compared with female non-fallers of the same age; (2) STS results did not differ significantly between female fallers and nonfallers; (3) female fallers demonstrated higher trait anxiety and a lower score of QoL, as compared with non-fallers.

The trend in STS test sway velocity suggested that changes in neurophysiological mechanisms for holding the posture in the conditions of a rapid rising from a sitting position would lead to the development of the geriatric "falls syndrome" in older women. Having analyzed the results of WA test, we observed the reduction in the walking rate of the fallers, which should be seen as a process of adaptive rearrangement in monotonous walking parameters, leading to the "falls syndrome". Our data suggested that significant changes in the components of a monotonous walk (speed, step length and width) in middle-aged and older women can be predictors of falls and reduced mobility.

Based on the data of TW test, we detected the changes in neurophysiological mechanisms for complex coordination movements in older female fallers. It was found that the changes in the TW test were a useful tool to identify older people predisposed to falls [5]. Thus, the results indicated the possibility of using this test for forecasting the development of the "falls syndrome" in older and senile patients with postural instability.

Analysis of SQT test results designated a decline in the capability to complex movements in older female fallers and changes of neurophysiological mechanisms responsible for complex motor acts in the elderly. Both are the factors of the "falls syndrome". Changes in the parameters of this test in middle-aged and older women may also be the consequence of reduction in their visual and vestibular information about controlling the balance; the ineffectiveness of the head and body stabilization in the course of walking, especially during fast turns; and dysfunction of the musculoskeletal system. Lee and Choi-Kwon [15] noted that vestibular changes in people $60 \mathrm{YO}$ and above were accompanied by decrease in QoL and increase in their anxiety level. Reduced muscle strength and extensor mechanism of the knee and feet increase the risk of falls. In order to prevent the development of geriatric "falls syndrome" and the risk of mobility deterioration in the elderly, health and fitness programs must be widely 
introduced, first of all, in order to strengthen their joints along with leg and back muscles [9].

Our study, results suggested no significant difference in state anxiety sensu Spielberger-Hanin between fallers and non-fallers. At the same time, the average values of these parameters in both groups were above 45 points, implying high level of anxiety. This finding indicated the presence of surveyed women with moderately reactive (state) anxiety, including female fallers. We also discovered aggravated trait anxiety in older female fallers. This means that development of high trait anxiety is a serious issue in older women living in the North of European Russia [7, 16-18]. Apparently, the prevalence of PsHCQOL and medium state anxiety in fallers showed that they had no indications of fearing the likelihood of falls. The results suggested that development of the "falls syndrome" in older women signposted a risk of their psychoemotional problems, promoting an emergence of depression and mental disorders.

The study proved that the loss of a postural stability function, along with increased risk of falls in middle-aged and older women have led to significant reduction in both PhHCQOL and MCQOL, thus having negative impact on health-related QoL $[9,19]$. It is known that development of falls in elderly women leads to a significant deterioration of their QoL.

Comparative analysis of SEA proved that, with age, in middleaged and older women, against the background of an objective assessment, the role of subjective assessment of their health status at a higher value would have a positive impact on their treatment and rehabilitation [7]. We established age-related disintegration of self-esteem in female fallers. It is known that SEA perception is a reflection of both human health state and life satisfaction, which essentially represent the quality of life. In addition, this indicator can be considered a criterion for evaluating the success of aging in people [4]. Successful aging is a physical, mental, social, psychological and socio-economic well-being, related to various factors. The latter include: low probability of disease or disability; the ability to learn; preservation of physical activity and fitness; continuing to work in retirement; active participation in a social life. All of these would help maintaining high QoL in the elderly people. Thus, the development of the "falls syndrome" among women 55-74 YO would negatively affect the process of successful aging.

On the basis of analyzing the RA, it can be concluded that an increase in RA in women 55-74 YO leads to reduced function of postural stability and development of a geriatric "falls syndrome". Thus, we revealed an RA increase in older fallers vs. non-fallers. Based on the characteristics of human RA, it can be concluded that development of the "falls syndrome" in elderly people should be considered an indicator of functional disorders and adaptive capability of the aging organism, leading to decrease in QoL [4, 20]. In addition, the results proved the need for creating the programs aimed at prevention of age-related conditions and accelerated aging in the elderly, specifically those living in the North of European Russia. Such programs would be beneficial to longevity and QoL $[4,19]$.

A reduction in the lift-up index (lifting the legs) in female fallers during the performance of complex movements, involving lifting and stepping over barrier was discovered. The results suggested that increased time of complex movements, being the first sign of problems with the balancing function, increased the risk of developing geriatric "falls syndrome" [9]. Also, a decrease in gait velocity among female fallers negatively affected their SEA perception.

Another implication of our results is that fallers decrease the speed of a monotonous walk with age. We found the connection between speed-time performance in the course of simple and complex gaits and SEA perception in older women predisposed to falls. The increased step width and reduced monotonous walk rate in female fallers, as well as decreased speed of the complex movement acts and adaptive capabilities, adversely affected SEA in this study. The results suggested that development of geriatric "falls syndrome" in women, who were overweight, have adversely affected the parameters of both monotonous and complex walking [20], and have significantly increased the risk of losing mobility and social independence.

Older women established the connection between the parameters of a monotonous walking and state anxiety, as well as the parameters of complex movement acts and trait anxiety. It could be assumed that development of higher levels of both state anxiety and trait anxiety would increase the risk of falls in older female fallers, and thereby increase the risk of losing mobility and social independence. Our study proved that an oscillating person's COG correlates with his or her mental and emotional state $[3,16]$.

The present study suggested that inflated state anxiety could lead to reduction in STS test lift-up index in fallers, as well as to increase in the WA test step width in the course of walking or performing complex coordination movements. These findings suggested that emergence of high trait anxiety could impair complex movement acts parameters in older women. The results showed that decreased functions of the knee joint extensor mechanism, along with decreased monotonous walk and complex movement acts, would adversely affect the QoL parameters [8, 14]. The parameters of walking in old and senile age would affect psychoemotional state and QoL [19]. Besides, the decline in muscle strength would adversely affect SEA perception in middleaged and older people. Thus, reduced SEA perception among older women has a negative impact on the process of successful aging. It should be considered the first sign of neurophysiological disorders and coping with aging.

It is well known that reducing complex coordination movements and complex motor acts in middle-aged and older women would increase environmental role as a risk factor for falls [12]. Thus, the development of a geriatric "falls syndrome" in elderly women should be seen as a paramount influence of environmental factors on human postural control, thereby having a negative impact on QoL and successful aging process. Our findings suggested that living environment and lifestyles have a significant impact on the conservation mechanisms of postural control in the course of aging. Furthermore, in the elderly, environmental role, as a factor of developing high trait anxiety, increases. Thus, it is vital to develop the measures aimed at creating a safe living environment for people of middle and old ages, which would favorably affect their QoL and the process of successful aging. Our findings reinforced an importance and effectiveness of exercise to strengthen leg muscles and joints, as well as back muscles, in older adults predisposed to falls as a criterion for QoL and successful aging process [14]. We established the relations between the speed-time performance of monotonous and complex modes of walking, and SEA perception in the elderly women with falls. Our study confirmed that parameters of walking in elderly and senile age women affected 
their psychoemotional state and QoL. In addition, the results showed that decline in muscle strength adversely affected SEA perception in middle-aged and older people $[4,16]$

\section{Conclusion}

Majority of mobility parameters, measured in the study, were lower in the group of female fallers compared with non-fallers. Middle-aged and older female fallers showed slower walking speed, while moving straight forward or during U-turns, and a wider step width in Tandem Walking. Fallers had higher levels of anxiety and more deteriorated mobility. We found no significant differences in STS test performance between the measured groups.

\section{Limitations}

Our study had some limitations that should be disclosed and discussed. The study participants were women living in the North of European Russia. Thus, it is necessary to compare our results with similar studies conducted in other regions, which are not subjected to extreme environmental conditions. All subjects in our study were relatively healthy, well-functioning older women 55-74 YO. Therefore, we recommend carrying out an analogous study on a group of younger and elderly women, and also comparing our results with the results of some prospective study conducted on both men and women.

\section{Conflict of interest}

Authors have no conflict of interest to disclose.

\section{Ethical approval}

All procedures performed in studies involving human participants were in accordance with the ethical standards of the institutional and/or national research committee and with the 1964 Declaration of Helsinki and its later amendments or comparable ethical standards.

\section{Acknolegments}

The study was carried out with the financial support of the Government of the Arkhangelsk Region (competition "Young Scientists of Pomerania") in the framework of the scientific project No. 10-2020 "Postural deficiency as an indicator of age-related compensatory-adaptive changes in the body of elderly people living in the Arctic Region of the Russian Federation".

\section{References}

1. Similä H, Immonen M, Merilahti J, Petäkoski-Hult T. Gait analysis and estimation of changes in fall risk factors. Conf Proc IEEE Eng Med Biol Soc 2015; 2015: 6939-6942. https://doi.org/10.1109/embc.2015.7319988.

2. Tideiksaar R. Falls in Older People: Prevention \& Management, 4th ed. Baltimore: Health Professions Press, 2010: 376 p. https://www.healthpropress.com/product/falls-in-older-people.

3. Ersoy Y, MacWalter RS, Durmus B, Altay ZE, Baysal O. Predictive effects of different clinical balance measures and the fear of falling on falls in postmenopausal women aged 50 years and over. Gerontology 2009; 55(6): 660-665. https://doi.org/10.1159/000235652.

4. Demin AV, Gydkov AB, Gribanov AV. Features of postural balance in elderly and old men. Human Ecology 2010; (12): 50-54. Russian. https://elibrary.ru/item.asp?id=15414586.
5. Beyaert C, Vasa R, Frykberg GE. Gait post-stroke: Pathophysiology and rehabilitation strategies. Neurophysiol Clin 2015; 45(4-5): 335355. https://doi.org/10.1016/i.neucli.2015.09.005

6. Pereira $\mathrm{CL}$, Baptista $\mathrm{F}$, Infante $\mathrm{P}$. Men older than 50 yrs are more likely to fall than women under similar conditions of health, body composition, and balance. Am J Phys Med Rehabil 2013; 92(12): 1095-1103. https://doi.org/10.1097/phm.0b013e31829b49eb.

7. Gydkov AB, Demin AV, Gribanov AV. Postural balance in elderly people in The North: monograph. Arkhangelsk: OAC "SOLTI". 2014: 190 p. Russian. https://elibrary.ru/item.asp?id=25220616.

8. O'Brien MK, Hidalgo-Araya MD, Mummidisetty $\mathrm{CK}$, Vallery $\mathrm{H}$, Ghaffari R, Rogers JA, et al. Augmenting Clinical Outcome Measures of Gait and Balance with a Single Inertial Sensor in Age-Ranged Healthy Adults. Sensors (Basel) 2019; 19(20): 4537. https://doi.org/10.3390/s19204537.

9. Lord SR, Sherrington C, Menz HB, Close JCT. Falls in Older People: Risk Factors and Strategies for Prevention. 2nd Ed. Cambridge: Cambridge University Press. 2007: 408 p. https://doi.org/10.1017/CBO9780511722233.

10. Batyrshina GR. Kinesiotherapeutic Technology "Aksarta" in the Posturological Control of Body Movements. Physical culture journal. Sport. Tourism. Motor recreation 2016; 1(3): 26-29. Russian. https://elibrary.ru/item.asp?id=27716714.

11. Skvortsov DV. Stabilometric study: short guide. Moscow: Mask. 2010: $176 \mathrm{p} . \quad$ Russian. https://www.biomera.ru/education/skvortsov dv.php.

12. Beyer $\mathrm{N}$, Simonsen $\mathrm{L}$, Bülow J, Lorenzen $\mathrm{T}$, Jensen $\mathrm{DV}$ et al. Old women with a recent fall history show improved muscle strength and function sustained for six months after finishing training. Aging Clin Exp Res 2007; 19(4): 300-309. https://doi.org/10.1007/bf03324706.

13. Pirker $W$, Katzenschlager R. Gait disorders in adults and the elderly: A clinical guide. Wien Klin Wochenschr 2017; 129(3-4): 81-95. https://doi.org/10.1007/s00508-016-1096-4.

14. Delafontaine A, Vialleron $T$, Fischer $M$, Laffaye $G$, Chèze L, Artico $R$ et al. Acute Effects of Whole-Body Vibration on the Postural Organization of Gait Initiation in Young Adults and Elderly: A Randomized Sham Intervention Study. Front Neurol 2019; 10: 1023. https://doi.org/10.3389/fneur.2019.01023

15. Lee HJ, Choi-Kwon S. Quality of life and the related factors in patients with dizziness. J Korean Acad Nurs 2009; 39(5): 751-758. Korean. https://doi.org/10.4040/jkan.2009.39.5.751.

16. Nakagawa HB, Ferraresi JR, Prata MG, Scheicher ME. Postura balance and functional independence of elderly people according to gender and age: cross-sectional study. Sao Paulo Med J 2017; 135(3): 260-265. $\quad$ https://doi.org/10.1590/15163180.2016 .0325280217$.

17. Payette $M-C$, Bélanger $C$, Léveillé $V$, Grenier S. Fall-Related Psychological Concerns and Anxiety among Community-Dwelling Older Adults: Systematic Review and Meta-Analysis. PLOS ONE 2016; 11(4): e0152848. https://doi.org/10.1371/journal.pone.0152848.

18. Murphy AT, Kravtsov S, Sangeux M, Rawicki B, New PW. Utilizing three dimensional clinical gait analysis to optimize mobility outcomes in incomplete spinal cord damage. Gait Posture 2019; 74: 53-59. https://doi.org/10.1016/j.gaitpost.2019.08.001.

19. Moroz TP, Demin AV, Volova AA. Peculiarities of life quality of old women having falls. Vestnik of Northern (Arctic) Federal University. Series "Medical and Biological Sciences" 2014; (3): 57-62. Russian. https://elibrary.ru/item.asp?id=22026830.

20. Garman CR, Franck CT, Nussbaum MA, Madigan ML. A bootstrapping method to assess the influence of age, obesity, gender, and gait speed on probability of tripping as a function of obstacle height. $J$ Biomech 2015; 48(6): https://doi.org/10.1016/j.jbiomech.2015.01.031. 
Authors:

Taisiya Petrovna Shiryaeva - Junior Researcher, M.V. Lomonosov Northern (Arctic) Federal University, Arkhangelsk, Russia; Laboratory Assistant, Institute of age physiology, Moscow, Russia. http://orcid.org/0000-00019458-3224.

Denis Michailovich Fedotov - PhD, Assistant Professor, Northern State Medical University, Arkhangelsk, Russia; Assistant Professor, M.V. Lomonosov Northern (Arctic) Federal University, Arkhangelsk, Russia. http://orcid.org/0000-0002-4067-8364.

Anatoliy Vladimirovich Gribanov - MD, DSc, Professor, M.V. Lomonosov Northern (Arctic) Federal University, Arkhangelsk, Russia; Institute of Age Physiology, Moscow, Russia. http://orcid.org/0000-0002-4714-6408.

Mikhail N. Pankov - PhD, Associate Professor, M.V. Lomonosov Northern (Arctic) Federal University, Arkhangelsk, Russia. http://orcid.org/00000003- 3293-5751.

Larisa F. Startseva - PhD, Assistant Professor, Academic Secretary, State Institution of Pharmaceutical Drugs and Good Practices, Moscow, Russia. http://orcid.org/0000-0002-9928-5362.

Sergey F. Bagretsov - Senior Lecturer, M.V. Lomonosov Northern (Arctic) Federal University, Arkhangelsk, Russia. http://orcid.org/0000-0003-0722$\underline{1597}$ 